\title{
Entropy of Automorphisms of AF Algebras
}

By

\author{
David E. Evans*
}

Here we consider a notion of topological entropy for automorphisms of $\mathrm{AF}$ algebras, based on a corresponding measure theoretic entropy of Connes and Størmer [6] for automorphisms of hyperfinite von Neumann algebras with an invariant trace. We show how to compute the entropy of the shift on certain AF algebras associated with topological Markov chains.

If $\mathscr{A}$ is a unital AF algebra, let $T(\mathscr{A})$ denote the normalized traces on $\mathscr{A}$. If $\phi \in T(\mathscr{A})$, and $B$ is a finite dimensional $C^{*}$-subalgebra of $\mathscr{A}$, let $E_{B}^{\phi}$ denote the conditional expectation of $\mathscr{A}$ onto $B$, relative to $\phi$. For simplicity, we will always only consider finite dimensional subalgebras, with the same unit as $\mathscr{A}$. If $n \in \mathbb{N}$, let $S_{n}$ denote the maps $x$ from $\mathbb{Z}^{n}$ into $\mathscr{A}_{+}$with finite support such that $\sum_{\alpha_{i} \in \mathbf{Z}} x\left(\alpha_{1}, \ldots, \alpha_{n}\right)=1$. For $1 \leq l \leq n, x \in S_{n}, \alpha \in \mathbb{Z}$, put

$$
x^{l}(\alpha)=\sum_{\alpha_{1}, \ldots, \alpha_{l-1}, \alpha_{l+1}, \ldots, \alpha_{n}} x\left(\alpha_{1}, \ldots, \alpha_{l-1}, \alpha, \alpha_{l+1}, \ldots, \alpha_{n}\right) .
$$

Also, let $\eta(x)=-x \log x$ for $x \in[0, \infty)$. Let $B_{1}, \ldots, B_{n}$ be finite dimensional subalgebras of $\mathscr{A}$. Then following [6], define:

$$
\begin{aligned}
H\left(B_{1}, \ldots, B_{n}\right)= & \sup _{\phi \in T(A)} \sup _{x \in S_{n}}\left\{\sum_{\alpha_{i} \in Z} \eta \phi x\left(\alpha_{1}, \ldots, \alpha_{n}\right)\right. \\
& \left.-\sum_{l=1}^{n} \sum_{\alpha \in \mathbf{Z}} \phi \eta E_{B_{l}}^{\phi}\left(x^{l}(\alpha)\right)\right\} .
\end{aligned}
$$

The proofs of [6] carry over to give that $H$ satisfies:

(A) $H\left(A_{1}, \ldots, A_{n}\right) \leq H\left(B_{1}, \ldots, B_{n}\right)$ if $A_{j} \subseteq B_{j}, j=1, \ldots, n$.

(B) $H\left(B_{1}, \ldots, B_{n}, B_{n+1}, \ldots, B_{n+m}\right) \leq H\left(B_{1}, \ldots, B_{n}\right)+H\left(B_{n \nmid 1}, \ldots, B_{n+m}\right)$.

(C) $B_{1}, \ldots, B_{n} \subseteq B \Rightarrow H\left(B_{1}, \ldots, B_{n}, B_{n+1}, \ldots, B_{n+m}\right) \leq H\left(B, B_{n+1}, \ldots, B_{n+m}\right)$

(D) For any family of minimal projections of $B,\left(e_{\alpha}\right)_{\alpha \in I}$ such that $\sum e_{\alpha}=1$, one has $H(B)=\sup _{\phi \in T(A)}\left(\sum_{\alpha \in I} \eta \phi\left(e_{\alpha}\right)\right)$.

(E) If $C^{*}\left(B_{1}, \ldots, B_{n}\right)$, the $C^{*}$-algebra generated by $B_{1}, \ldots, B_{n}$ is generated by

Communicated by H. Araki, October 28, 1981.

* Mathematics Institute, University of Warwick, Coventry CV4 7AL, England. 
pairwise commuting $C^{*}$-subalgebras $P_{j}$ of $B_{j}$, then $H\left(B_{1}, \ldots, B_{n}\right)=$ $H\left(C^{*}\left(B_{1}, \ldots, B_{n}\right)\right)$.

If $\sigma$ is an automorphism of $\mathscr{A}$, and $B$ a finite dimensional $C^{*}$-subalgebra of $\mathscr{A}$, let $H(B, \sigma)=\lim _{k \rightarrow \infty} H\left(B, \sigma B, \ldots, \sigma^{k} B\right) / \mathrm{k}$. Then define the (topological) entropy of $\sigma$ to be $H(\sigma)=\sup H(B, \sigma)$, where the supremum is taken over all finite dimensional subalgebras $B$ of $\mathscr{A}$. If $\mathscr{A}$ is also commutative, i.e. $\mathscr{A}=C(X)$, where $X$ is a totally disconnected compact Hausdorff space, and if $B$ is a finite dimensional subalgebra with minimal projections $\left(e_{\alpha}\right)_{\alpha \in I}$, and $\sum e_{\alpha}=1$, then by $D, H(B)=\sup _{\phi \in T(A)}\left\{\sum_{\alpha} \eta \phi\left(e_{\alpha}\right)\right\}=\log |I|$. It then becomes clear from $E$ that if $\sigma$ is an automorphism induced by a homeomorphism $\hat{\sigma}$ of $X$, then $H(\sigma)$ is the same as the classical topological entropy of $\hat{\sigma},[1]$. Moreover, suppose $\sigma$ is an automorphism of an AF algebra $\mathscr{A}$ with invariant trace $\phi$. Then let $(\pi, H, \xi)$ be the GNS decomposition of $\phi$, and let $\tilde{\sigma}$ be the induced automorphism of $\widetilde{A}=\pi(\mathscr{A})^{\prime \prime}$, with invariant faithful vector trace $\tilde{\phi}(\cdot)=\langle(\cdot) \xi, \xi\rangle$. Then it follows from the Kolmogorov-Sinai theorem of [6] that $H(\sigma) \geq H_{\tilde{\phi}}(\tilde{\sigma})$, the latter being the measure-theoretic entropy of $\tilde{\sigma}$ on $\tilde{\mathscr{A}}$ with respect to $\tilde{\phi}$.

Proposition 1. If $\mathscr{A}=\overline{\cup B_{n}}$, with $B_{n} \subseteq B_{n+1}$ finite dimensional $C^{*}$ subalgebras, and $\sigma$ an automorphism of $A$, then $H(\sigma)=\lim _{n \rightarrow \infty} H\left(B_{n}, \sigma\right)$.

Proof. Define for $B$ and $C$ finite dimensional $C^{*}$-subalgebras of $\mathscr{A}$ :

$$
H(B \mid C)=\sup _{\phi \in T(A)} \sup _{x \in S_{1}}\left(\sum_{i}\left\{\phi \eta E_{C}^{\phi} x(i)-\phi \eta E_{B}^{\phi}(x(i))\right\}\right) .
$$

Say that $B \stackrel{\delta}{\complement} C$ for $\delta>0$, if for each $b$ in $B$, there exists $c$ in $C$ such that $\|b-c\|$ $\leq \delta\|b\|$. Claim that if $B$ is fixed and $\varepsilon>0$, there exists $\delta>0$, such that if $B \stackrel{\delta}{\subset} C$, then $H(B \mid C)<\varepsilon$. An examination of the proof of [6, Lemma 10] shows that if $B$ is fixed and $\varepsilon>0$, then there exists $\varepsilon^{\prime}>0$, such that if $v$ is a unitary in $\mathscr{A}$ with $\|v-1\|<\varepsilon^{\prime}$, then $H\left(B \mid v B v^{*}\right)<\varepsilon$. Now by [5, Theorem 6.4], given $\varepsilon^{\prime}>0$, there exists $\delta>0$ such that if $B \stackrel{\delta}{\subset} C$, then there exists a unitary $v$ in $\mathscr{A}$ such that $v B v^{*} \subseteq C$ and $\|v-1\|<\varepsilon^{\prime}$. Thus for $B \stackrel{\delta}{\subset} C$,

$$
\begin{aligned}
H(B \mid C) & \leq H\left(B \mid v B v^{*}\right)+H\left(v B v^{*} \mid C\right) \text { from definitions } \\
& \leq H\left(B \mid v B v^{*}\right) \text { as } H \text { is increasing in first variable } \\
& <\varepsilon .
\end{aligned}
$$

Thus if $B$ is any finite dimensional $C^{*}$-subalgebra of $\mathscr{A}$, choose $B_{n}$ such that $B \stackrel{\delta}{\subset} B_{n}, H\left(B \mid B_{n}\right)<\varepsilon$, and

$$
H(B, \sigma)=\lim _{k} \frac{1}{k} H\left(B, \sigma B, \ldots, \sigma^{k} B\right)
$$




$$
\begin{aligned}
& \leq \lim _{k} \frac{1}{k} H\left(B_{n}, \sigma B_{n}, \ldots, \sigma^{k} B_{n}\right)+\varlimsup_{k} \frac{1}{k} \sum_{j=0}^{k} H\left(\sigma^{j}(B) \mid \sigma^{j}\left(B_{n}\right)\right) \\
& \quad \text { from definitions } \\
& \leq H\left(B_{n}, \sigma\right)+\varepsilon .
\end{aligned}
$$

The proposition follows.

Let $A$ be an aperiodic $n \times n$ matrix with entries in $\{0,1\}$ and let $\Sigma=\{1,2, \ldots, n\}$. Let $\mathscr{F}^{A}$ be the associated two sided unital AF algebra with dimension group $\lim \left(\mathbb{Z}^{n^{2}}, A \times A^{t}\right)$ and dimension range

$$
\lim _{m \rightarrow \infty} \Gamma_{m}, \text { where } \Gamma_{m}=\left\{x \in \mathbb{Z}^{n^{2}} ; 0 \leq x \leq\left(A \times A^{t}\right)^{m}(A)\right\},
$$

and $\mathbf{Z}^{n^{2}}$ is given the simplicial ordering (see $[10,7,9]$ ). Thus let $X_{A}$ denote the compact space $\left\{\left(x_{i}\right) \in \Sigma^{z}: A\left(x_{i}, x_{i+1}\right)=1\right\}$, and $\mathscr{G}$ be the group of all uniformly finite dimensional homeomorphisms of $X_{A}$ which change only finitely many coordinates. Let $\mathscr{A}$ denote the $C^{*}$-crossed product $C^{*}\left(X_{A}, \mathscr{G}\right)$ and let $U$ denote the canonical representation of $\mathscr{G}$ in $\mathscr{A}$. If $u$ is a uniformly finite dimensional homeomorphism of a compact open subset $B$ onto a compact open subset $C$ of $X_{A}$, let $\tilde{u}$ denote the corresponding partial isometry in $\mathscr{A}$ with initial projection $P(B)$, the characteristic function of $B$ in $C\left(X_{A}\right)$, and final projection $P(C)$. Let $\mathscr{I}$ denote the ideal generated by $U(g) P(B)-U(h) P(B)$, where $B$ is a compact open subset of $X_{A}$ and where $g, h \in \mathscr{G}$ agree on $\mathscr{B}$. Then let $\mathscr{F}^{A}=\mathscr{A} / \mathscr{I}$. The shift $\sigma$ on $X_{A}$, normalises $\mathscr{G}$ and induces an automorphism of $\mathscr{F}^{A}$ also denoted by $\sigma$. The AF algebra $\mathscr{F}^{A}$ can be described as follows:

Let $\mathscr{M}_{r s}^{i j}=\left\{\left(i_{-r}, \ldots, i_{-1}, i_{0}, \ldots, i_{s}\right): i_{k} \in \Sigma, A\left(i_{k}, i_{k \perp 1}\right)=1, i_{-r}=i, i_{s}=j\right\}$ for $i, j \in \Sigma, \quad r, s \in \mathbb{N}$. Let $\mathscr{M}_{r s}=\bigcup_{i, j} \mathscr{M}_{r s}^{i j}, \mathscr{M}_{s}^{i j}=\mathscr{M}_{s s}^{i j}, \mathscr{M}_{s}=\mathscr{M}_{s s}$. If $\mu \in \mathscr{M}_{r s}^{i j}$, let $Z(\mu)=\left\{x \in X_{A}:\left(x_{i}\right)_{-r}^{s}=\mu\right\}$. If $\mu, v \in \mathscr{M}_{r s}^{i j}$, let $u_{\mu v}$ denote the u.f.d. homeomorphism of $Z(v)$ onto $Z(\mu)$ given by $\left(u_{\mu v}(x)\right)_{i}=x_{i}$ if $i<-r$, or $i>s$, and put $e_{\mu v}$ $=\tilde{u}_{\mu v}, e_{\mu}=e_{\mu \mu}$. Then $\left\{e_{\mu v}: \mu, v \in \mathscr{M}_{s}^{i j}\right\}$ are matrix units and generate a finite dimensional matrix algebra $M_{s}^{i j}$. Then if $M_{s}=\oplus_{i j} M_{s}^{i j}, M_{s} \subseteq M_{s+1}, \mathscr{F}^{A}$ $=\bar{\cup} M_{s}$, and the embedding of $M_{s}$ in $M_{s+1}$ is given by $A \times A^{t}$. The gauge action of $\stackrel{s}{T^{n}}$ on $\mathscr{F}^{A}$ is defined as follows. If $t=\left(t_{i}\right)_{i=1}^{n} \in \mathbb{C}^{n}$, and $\mu=\left(i_{-s}, \ldots, i_{s}\right) \in \mathscr{M}_{s}$ let $t_{\mu}=t_{i_{-s}} \ldots t_{i_{s}} \in \mathbb{C}$. Then let $\mathbb{T}^{n}$ act on $\mathscr{F}^{A}$ by $t: e_{\mu v} \rightarrow t_{\mu} \bar{t}_{v} e_{\mu v}$, for $\mu, v \in \mathscr{M}_{s}, t \in \mathbb{T}^{n}$. Let $\mathscr{C}^{A}$ denote the gauge invariant or observable algebra $\left(\mathscr{F}^{A}\right)^{T^{n}}$, and let $\sigma_{0}$ denote the restriction of $\sigma$ to $\mathscr{C}^{A}$. Then $\mathscr{C}^{A}$ is an $A F$ algebra, which can be described as follows $[3,4]$ : If $\left(r_{i}\right)_{i=1}^{n} \in \mathbb{N}^{n}$, with $\sum_{i=1}^{n} r_{l}=2 s+1$, let $\Lambda_{i j}^{s}\left(r_{1} \ldots, r_{n}\right)$ denote the set of $\mu$ in $\mathscr{M}_{s}^{i j}$, such that $k$ in $\Sigma$ occurs in $\mu$ exactly $r_{k}$ times. 
Then let $N_{i j}^{s}\left(r_{1}, \ldots, r_{n}\right)$ denote the matrix algebra generated by $\left\{e_{\mu \nu}: \mu, v \in\right.$ $\left.\Lambda_{i j}^{s}\left(r_{1}, \ldots, r_{n}\right)\right\}$, and put $N_{s}=\underset{\substack{i, j \\ r_{1}, \ldots, r_{n}}}{\oplus} N_{i j}^{s}\left(r_{1}, \ldots, r_{n}\right)$. Then $N_{s}=\left(M_{s}\right)^{\mathbf{T}^{n}}, N_{s} \subseteq N_{s+1}$, and $\mathscr{C}^{A}=\overline{\bigcup_{s} N_{s}}$.

We first show the following which may be of independent interest. The use of the theory of the chemical potential for the GICAR algebra was first advocated by G. A. Elliott.

Proposition 2. If $\phi$ is a $\sigma_{0}$ invariant, faithful extremal tracial state on $\mathscr{C}^{A}$, then there exist $\rho_{i}, u_{i}, v_{i}, \theta$ in $(0, \infty)$ with $\sum \rho_{r} A(r, i) u_{r}=\theta u_{i}, \sum A(j, t) \rho_{t} v_{t}$ $=\theta v_{j}$ such that

(F) $\phi\left(e_{\mu v}\right)=\rho_{\mu} u_{i} v_{j} / \theta^{-2 s} \delta_{\mu v}$, for $\mu, v \in \Lambda_{i j}^{s}\left(r_{1}, \ldots, r_{n}\right)$ where $\rho_{\mu}=\rho_{1}^{r_{1}} \rho_{2}^{r_{2}} \cdots \rho_{n}^{r_{n}}$.

Proof. It is easy to check that $\sigma$ is norm asymptotically abelian on $\mathscr{F} A$, i.e. $\lim _{m \rightarrow \infty}\left\|\sigma^{m}(a) b-b \sigma^{m}(a)\right\|=0$, for all $a, b$ in $\mathscr{F}^{A}$. Hence by [2], there exist $h_{1}, \ldots, h_{n}$ in $\mathbf{R}$ such that if $\gamma(t)=\left(\exp \left(i h_{j} t\right)\right)_{j=1}^{n} \in T^{n}$, for $t \in \mathbb{R}$, then $\phi$ is the restriction of a state $\psi$ on $\mathscr{F}^{A}$ which is KMS with respect to the gauge action $\{\gamma(t): t \in \mathbf{R}\}$ on $\mathscr{F}^{A}$ at inverse temperature +1 . By uniqueness of KMS states on full matrix algebras, there exist scalars $a_{i j}^{s}>0$ such htat

$$
\psi e_{\mu \nu}=\rho_{\mu} a_{i j}^{s} \delta_{\mu \nu}, \quad \text { for } \quad \mu, v \in \mathscr{M}_{s}^{i j}
$$

and where $\left(\rho_{i}\right)=\left(\exp \left(-h_{i}\right)\right) \in(0, \infty)^{n}$. Then by considering the embedding of $M_{s}$ in $M_{s+1}$,

$$
\sum_{r, t} A(r, i) A(j, t) \rho_{r} \rho_{t} a_{r t}^{s+1}=a_{i j}^{s} .
$$

The $n^{2} \times n^{2}$ matrix $\left\{A(r, i) A(j, t) \rho_{r} \rho_{t}:(i, j),(r, t) \in \Sigma^{2}\right\}$ is irreducible, and so by the theorem of Perron-Frobenius, it possesses a strictly positive eigenvector, say $\left\{b(i, j):(i, j) \in \Sigma^{2}\right\}$ which is unique up to scalar multiplication. If $\rho$ denotes the diagonal matrix $\left[\rho_{i} \delta_{i j}\right]$, let $\theta$ denote the spectral radius of $\rho A$ which is also the spectral radius of $A \rho$, as $\sigma(\rho A) \cup\{0\}=\sigma(A \rho) \cup\{0\}$. Then let $\left(u_{i}\right)$ and $\left(v_{i}\right)$ be the (essentially) unique strictly positive eigenvectors satisfying $\sum \rho_{r} A(r, i) u_{r}$ $=\theta u_{i}, \sum A(j, t) \rho_{t} v_{t}=\theta v_{j}$. Then $\sum_{r, t} A(r, i) A(j, t) \rho_{r} \rho_{t} u_{r} v_{t}=\theta^{2} u_{i} v_{j}$. Thus by uniqueness, and rescaling if necessary, $b(i, j)=u_{i} v_{j}$. Then by [9, Theorem 6.1], extended to the case of irreducible matrices, with not necessarily integral entries, it follows that $a_{i j}^{s}=b(i, j) / \theta^{-2 s}$, after rescaling $b$ if necessary. Thus $\psi\left(e_{\psi v}\right)$ $=\rho_{\mu} u_{i} v_{j} / \theta^{-2 s} \delta_{\mu v}$, for $\mu, v \in \mathscr{M}_{s}^{i j}$.

Theorem. $H\left(\sigma_{0}\right)=\log \lambda$, where $\lambda$ is the spectral radius of $A$. 
Proof. Let $B=C^{*}\left(e_{\mu 1}: e_{\mu \nu} \in \mathscr{C}^{A}, \mu, v \in \mathscr{M}_{s, s+k}^{i j}, \quad(i, j) \in \Sigma^{2}\right)$ for $s, k \in \mathbb{N}$. Then $\sigma_{0}^{i}\left(N_{s}\right) \subset B$ for $i=0,1,2, \ldots, k$. Then

$$
\begin{gathered}
H\left(N_{s}, \sigma_{0}\left(N_{s}\right), \ldots, \sigma_{0}^{k}\left(N_{s}\right)\right) \leq H(B) \quad \text { by } \quad C \\
\quad=\sup _{\phi \in T(\mathscr{C} A)}\left\{\sum \eta \phi\left(e_{\mu}\right): \mu \in \mathscr{M}_{s, s+k}\right\} \quad \text { by } \quad D \\
\quad \leq \log \left|\mathscr{M}_{s, s+k}\right|=\log \left(\sum_{i, j} A^{2 s+k+1}(i, j)\right) .
\end{gathered}
$$

Hence $H\left(N_{s}, \sigma\right) \leq \lim _{k \rightarrow \infty} \frac{1}{k} \log \left(\sum A^{2 s+k+1}(i, j)\right)=\log \lambda$, by a computation [8, p. 128] of the classical entropy of $\sigma$ on $X_{A}$. Taking the partition of unity given by

$$
\mu_{0}, \mu_{1}, \ldots, \mu_{k} \longrightarrow e_{\mu_{0}} \sigma_{0}\left(e_{\mu_{1}}\right) \cdots \sigma_{0}^{k}\left(e_{\mu_{k}}\right), \quad \mu_{i} \in \mathscr{M}_{s},
$$

we have

$$
\begin{aligned}
H\left(N_{s}, \sigma_{0}\left(N_{s}\right), \ldots, \sigma_{0}^{k}\left(N_{s}\right)\right) & \geq \sup _{\phi \in T\left(C^{A}\right)}\left\{\sum_{\mu_{j}} \eta \phi\left(e_{\mu_{0}} \sigma_{0}\left(e_{\mu_{1}}\right) \cdots \sigma_{0}^{k}\left(e_{\mu_{k}}\right)\right)\right\} \\
& =\sup _{\phi \in T\left(C^{A}\right)}\left\{\sum_{\mu} \eta \phi\left(e_{\mu}\right): \mu \in \mathscr{M}_{s, s+k}\right\} .
\end{aligned}
$$

Let $\phi_{0}$ be the (normalised) trace on $\mathscr{C}^{A}$ given by $F$, with $\rho_{i}=1 / n, i \in \Sigma$, and where $u_{i}, v_{i}>0$ satisfy $\sum A(r, i) u_{r}=\lambda u_{i}, \sum A(j, t) v_{t}=\lambda v_{j}$, and where $\lambda$ is the spectral radius of $A$. Then again by considering the entropy of $\sigma$ on $X_{A}$, with respect to the invariant measure $\left.\phi_{0}\right|_{\mathscr{C}\left(X_{A}\right)}[8$, p. 69, 129] it is seen that

$$
\left.H\left(N_{s}, \sigma\right) \geq \varlimsup_{k} \frac{1}{k}\left(\sum_{n} \eta \phi_{0}\left(e_{\mu}\right)\right) \text { (summation over } \mu \in \mathscr{M}_{s, s+k}\right)=\log \lambda .
$$

The result follows from Proposition 1.

Remark 1. The theorem shows that the entropy of $\sigma_{0}$ is the same as the classical entropy of its restriction to $X_{A}[11]$. Similarly, $H(\sigma)=\log \lambda$.

Remark 2. Consider the case of the full $n$-shift, where $A(i, j) \equiv 1$. Then every extremal trace corresponds to $\Delta=\left\{\left(\rho_{i}\right)_{i=1}^{n}=\rho_{i} \geq 0, \sum \rho_{i}=1\right.$ and all are invariant under $\sigma_{0}$. If $\left(\rho_{\iota}\right) \in \Delta$, the corresponding trace is given by $e_{\mu \nu} \rightarrow \rho_{\mu} \delta_{\mu v}$. This is essentially a classical Hausdorff moment problem. Given $\left\{a_{r_{1} \ldots r_{n}}^{s} \geq 0: s=1,2\right.$, $\left.\ldots, r_{i} \in \mathbf{N}, \sum_{i=1}^{n} r_{i}=s\right\}$ such that $a_{r_{1} \ldots r_{n}}^{s}=\sum_{i=1}^{n} a_{r_{1} \ldots r_{i}+1 \ldots r_{n}}^{s+1}$, there exists a finite measure $\theta$ on $\Delta$ such that $a_{r_{1} \ldots r_{n}}^{s}=\int_{\Delta} \rho_{1}^{r_{1}} \cdots \rho_{n}^{r_{n}} d \theta\left(\rho_{1}, \ldots, \rho_{n}\right)$. This can be proved by classical methods (see e.g. [13, Theorems $1.5,1.6]$ for the cases $n=2,3$ ) or alternatively by looking at $K_{0}\left(\mathscr{C}^{A}\right)$. Now $K_{0}\left(\mathscr{C}^{A}\right)$ can be identified with the ring $\mathbb{Z}\left[t_{1}, \ldots, t_{n-1}\right]$ of polynomials in indeterminates $t_{1}, \ldots, t_{n-1}$ with integer coefficients, such that a non-zero $p$ in $\mathbb{Z}\left[t_{1}, \ldots, t_{n-1}\right]$ lies in the positive cone if 
and only if $p\left(\rho_{1}, \ldots, \rho_{n-1}\right)>0$ whenever $\left(\rho_{1}, \ldots, \rho_{n-1}, 1-\sum_{i=1}^{n-1} \rho_{i}\right)$ lies in the interior of $\Delta$, [12]. Then if $\phi$ is the trace on $\mathscr{C}^{A}$ corresponding to $\left(\rho_{1}, \ldots, \rho_{n}\right) \in \Delta$, it follows as in the above theorem that $H_{\tilde{\phi}}\left(\tilde{\sigma}_{0}\right)=-\sum_{i=1}^{n} \rho_{\imath} \log \rho_{i}$, (cf. the Bernoulli shift defined by $\left\{\rho_{1}, \ldots, \rho_{n}\right\}$ in the sense of $\left[6\right.$, Definition 2]). Thus $H_{\tilde{\phi}}\left(\tilde{\sigma}_{0}\right)$ $<H\left(\sigma_{0}\right)$, unless $\rho_{i} \equiv 1 / n$, or $\phi=\phi_{0}$. Thus by convexity, $H_{\tilde{\phi}}\left(\tilde{\sigma}_{0}\right)<H\left(\sigma_{0}\right)$, for any trace $\phi$ on $\mathscr{C}^{A}$ distinct from $\phi_{0}$. Hence $\phi_{0}$ is the unique trace with maximal entropy. In the more general situation of the main theorem, it can be shown that $H_{\tilde{\phi}}\left(\tilde{\sigma}_{0}\right)<H\left(\sigma_{0}\right)$ if $\phi$ is any trace satisfying the conditions of Proposition 2, unless $\phi=\phi_{0}$, the canonical trace used to compute the topological entropy.

Remark 3. Let $A\left(l_{2}\right)=C^{*}\left(a(h): h \in l_{2}\right)$ be the CAR algebra generated by a representation $a$ of the canonical anti-commutation relations of the complex Hilbert space $l_{2}$. Let $u$ denote the two-sided shift on $I_{2}$ and $\theta$ be the associated Bogoliubov automorphism of $A\left(l_{2}\right)$, and $\theta_{0}$ its restriction to the current algebra. Then as in the proof of the Theorem, $H(\theta)=\log 2=H\left(\theta_{0}\right)$, but it is unclear whether $\theta$, (respectively $\theta_{0}$ ) is conjugate to the shift on $\bigotimes_{-\infty}^{\infty} M_{2}$ (respectively the restriction of the shift to $\left(\bigotimes_{-\infty}^{\infty} M_{2}\right)^{\mathbf{T}^{2}}$, with $\mathbb{T}^{2}$ acting by the usual product action).

\section{Acknowledgements}

This work was completed during a visit to the Institute of Mathematics, University of Copenhagen. I am very grateful to Erik Christensen, George Elliott, Dorte Olesen and Gert Kjærgard Pedersen for their warm hospitality, and the Royal Society for their generous support.

\section{References}

[1] R. L. Adler, A. G. Konheim and M. H. McAndrew, Topological entropy, Trans. Amer. Math. Soc., 114 (1965), 309-319.

[2] H. Araki, R. Haag, D. Kastler and M. Takesaki, Extension of states and chemical potential, Commun. Math. Phys., 53 (1977), 97-134.

[3] O. Bratteli, Inductive limits of finite dimensional $C^{*}$-algebras, Trans. Amer. Math. Soc., 191 (1972), 195-234.

[4] - Crossed products of UHF algebras by product type actions, Duke Math. $J ., 46$ (1979), 1-23.

[5] E. Christensen, Near inclusions of $C^{*}$-algebras. Acta Math., 144 (1980), 249-265.

[6] A. Connes and E. Størmer, Entropy of automorphisms of $\mathrm{II}_{1}$ von Neumann algebras, Acta Math., 134 (1975), 289-306. 
[ 7] J. Cuntz and W. Krieger, A class of $C^{*}$-algebras and topological Markov chains, Inventiones Math., 56 (1980), 251-268.

[ 8 ] M. Denker, C. Grillenberger and K. Sigmund, Ergodic theory on compact spaces, Springer Lecture Notes in Mathematics, 527, 1976.

[9] E. G. Effros, Dimensions and $C^{*}$-algebras, Regional Conference Series in Mathematics, Amer. Math. Soc., to appear.

[10] W. Krieger. On dimension functions and topological Markov Chains, Inventiones Math. 56 (1980), 239-250.

[11] W. Parry, Intrinsic Markov Chains, Trans. Amer. Math. Soc., 112 (1964), 55-66.

[12] J. Renault, A groupoid approach to $C^{*}$-algebras, Springer Lecture Notes in Mathematics., 793, 1980.

[13] J. A. Shohat and J. D. Tamarkin, The problem of moments, Mathematical Surveys 1, American Math. Soc. 1963. 
